\title{
A study of remainders of topological groups
}

by

\author{
A. V. Arhangel'skii (Athens, $\mathrm{OH})$
}

\begin{abstract}
Some duality theorems relating properties of topological groups to properties of their remainders are established. It is shown that no Dowker space can be a remainder of a topological group. Perfect normality of a remainder of a topological group is consistently equivalent to hereditary Lindelöfness of this remainder. No $L$-space can be a remainder of a non-locally compact topological group. Normality is equivalent to collectionwise normality for remainders of topological groups. If a non-locally compact topological group $G$ has a hereditarily Lindelöf remainder, then $G$ is separable and metrizable. We also present several other criteria for a topological group $G$ to be separable and metrizable. Two of them are of general nature and depend heavily on a new criterion for Lindelöfness of a topological group in terms of remainders. One of them generalizes a theorem of the author [Topology Appl. 150 (2005)] as follows: a topological group $G$ is separable and metrizable if and only if some remainder of $G$ has locally a $G_{\delta}$-diagonal. We also study how close are the topological properties of topological groups that have homeomorphic remainders.
\end{abstract}

1. Introduction: some recent results. By a "space" we understand a Tikhonov topological space. By a remainder of a space $X$ we mean the subspace $b X \backslash X$ of a Hausdorff compactification $b X$ of $X$. We study how properties of remainders of topological groups are related to the topological properties of the groups. An important part of our techniques is the famous classical result of M. Henriksen and J. Isbell [13]:

TheOREM 1.1. A space $X$ is of countable type if and only if the remainder in any (or some) compactification of $X$ is Lindelöf.

Recall that a space $X$ is of countable type if every compact subspace $P$ of $X$ is contained in a compact subspace $F \subset X$ which has a countable base of open neighbourhoods in $X$. All metrizable spaces and all locally compact spaces, as well as all Čech-complete spaces, are of countable type [1]. It

2000 Mathematics Subject Classification: Primary 54H11, 54D40; Secondary 54A25.

Key words and phrases: remainder, compactification, topological group, $p$-space, Lindelöf $p$-space, metrizability, countable type, Lindelöf space, pseudocompact space, $\pi$-base, subcountable type, Dowker space. 
follows from Theorem 1.1 that every remainder of a metrizable space is Lindelöf, and hence paracompact.

A series of results on remainders of topological groups have been obtained in [5]-[8]. They show that remainders of topological groups are much more sensitive to the topological properties of groups than the remainders of topological spaces are in general. In particular, a Dichotomy Theorem, saying that every remainder of any topological group is either pseudocompact or Lindelöf, has been established in [8]. Using this theorem, we conclude that no Dowker space can be a remainder of a topological group, and that normality is equivalent to collectionwise normality in remainders of topological groups. Perfect normality of a remainder $Y$ of a topological group is shown to be consistently equivalent to hereditary Lindelöfness of $Y$. We also provide some new characterizations of a few popular classes of topological groups by certain properties of their remainders. In particular, we improve a theorem from [6] in the following way: a non-locally compact topological group $G$ is separable and metrizable if (and only if) some remainder $Y$ of $G$ has locally a $G_{\delta^{-}}$-diagonal. A few open problems are formulated.

Recall that paracompact p-spaces [1] can be characterized as preimages of metrizable spaces under perfect mappings. A Lindelöf p-space is a preimage of a separable metrizable space under a perfect mapping. For the definition of a $p$-space see [1], where it was shown that every $p$-space is of countable type, and that every metrizable space is a $p$-space.

Clearly, every separable metrizable space has a separable metrizable remainder. Here is a parallel result from [6]:

TheOREM 1.2. If $X$ is a Lindelöf p-space, then every remainder of $X$ is a Lindelöf p-space.

A $\pi$-base of a space $X$ at a subset $F$ of $X$ is a family $\gamma$ of non-empty open subsets of $X$ such that every open neighbourhood of $F$ contains at least one element of $\gamma$. It is a well known theorem of Birkhoff and Kakutani that every first countable topological group is metrizable (see, for example, [11]). We repeatedly use the following generalization of this statement, found in [3].

Proposition 1.3. If a topological group $G$ has a countable $\pi$-base at some point a, then $G$ is metrizable.

Proof. Since the space $G$ is homogeneous, we may assume that $G$ has a countable $\pi$-base $\eta$ at the neutral element $e$ of $G$. Then, clearly, the family $\xi=\left\{V V^{-1}: V \in \eta\right\}$ is a countable base of $G$ at $e$. Consequently, $G$ is first countable, and hence metrizable.

The following results were obtained in [8]. Since the present article is, in fact, a continuation of [8], we have to list quite a few of them. 
Lemma $1.4([8])$. Suppose that $X$ is a nowhere locally compact space, and that $b X$ is a compactification of $X$ such that the remainder $Y=b X \backslash X$ is not pseudocompact. Then there exists a non-empty compact subspace $F$ of $X$ which has a countable $\pi$-base in $X$.

Lemma 1.5 ([8]). Suppose that $G$ is a topological group, and that $F$ is a non-empty compact subspace of $G$ such that $G$ has a countable $\pi$-base at $F$. Then $G$ is a paracompact p-space.

TheOREM 1.6 ([8]). For any topological group $G$, either every remainder of $G$ is pseudocompact or every remainder of $G$ is Lindelöf.

The next theorem [6] immediately follows from Theorem 1.6 and Lemmas 1.4 and 1.5 .

THEOREM 1.7. A topological group $G$ has a Lindelöf remainder if and only if $G$ is a paracompact p-space (and then all remainders of $G$ are Lindelöf).

Here is yet another result from [8]:

Theorem 1.8. Suppose that $G$ is a topological group, and $Y=b G \backslash G$ is the remainder of $G$ in a compactification of $G$. Then the following conditions are equivalent:

(1) $Y$ is Dieudonné complete.

(2) $Y$ is paracompact.

(3) Every remainder of $G$ is Lindelöf.

(4) $G$ is a paracompact p-space.

We also repeatedly use the following simple fact: if a topological group $G$ is not locally compact, then the remainder $Y$ of $G$ in any compactification $b G$ is dense in $b G$. This occurs because every non-locally compact topological group is nowhere locally compact.

Several results of this article were announced without proofs in [9]. Now the proofs are provided, along with the proofs of many new results.

2. Some duality theorems involving remainders. We start this section with the following corollary from the Dichotomy Theorem saying that every remainder of an arbitrary topological group $G$ has a certain compactness type property.

THEOREM 2.1. If $Y$ is a remainder of an arbitrary topological group $G$, then every discrete family of non-empty open subsets of the space $Y$ is countable, that is, $Y$ is pseudo- $\omega_{1}$-compact.

Proof. Indeed, both Lindelöf spaces and pseudocompact spaces satisfy the conclusion of the theorem. It remains to apply the Dichotomy Theorem. 
Let us call a space $X \pi$-metrizable if it has a $\sigma$-discrete $\pi$-base. Here is a curious corollary from Theorem 2.1:

COROLLARY 2.2. If a non-locally compact topological group $G$ has a $\pi$ metrizable remainder $Y$, then $G$ is separable and metrizable.

Proof. Indeed, it follows from Theorem 2.1 that $Y$ has a countable $\pi$ base. Since $G$ is a non-locally compact topological group, this implies that $G$ has a countable $\pi$-base. Hence, $G$ is separable and metrizable [2].

We will now establish a result related to Lemma 1.4; we will need it to prove some special versions of Theorem 1.7.

Lemma 2.3. Suppose that $B=X \cup Y$, where $B$ is a compact space, and $X, Y$ are dense nowhere locally compact subspaces of $B$. Suppose also that every compact subset of $Y$ is contained in a compact $G_{\delta}$-subset of $Y$. Then every locally finite (in $X)$ family $\gamma=\left\{U_{\alpha}: \alpha \in A\right\}$ of non-empty open subsets of the space $X$ is countable (that is, the set $A$ is countable).

Proof. Let $F$ be the set of all accumulation points of $\gamma$ in $B$. Clearly, $F$ is compact, and $F \subset Y$, since $\gamma$ is locally finite in $X$.

For each $\alpha \in A$ we fix an open subset $W_{\alpha}$ of $B$ such that $U_{\alpha}=X \cap W_{\alpha}$, and put $V_{\alpha}=Y \cap W_{\alpha}$.

Since $X$ is dense in $B, F$ is the set of all accumulation points of the family $\xi=\left\{W_{\alpha}: \alpha \in A\right\}$ in $B$.

Let us also consider the family $\eta=\left\{V_{\alpha}: \alpha \in A\right\}$ of open subsets of $Y$. Since $Y$ is dense in $B$, all elements of $\eta$ are non-empty.

Claim. Every open neighbourhood $O F$ of $F$ in $Y$ contains all but finitely many elements of $\eta$.

To verify this, take any open neighbourhood $H$ of $F$ in $B$ such that $O F=H \cap Y$. Since $F$ is compact, there exists an open neighbourhood $E$ of $F$ in $B$ such that the closure $\bar{E}$ of $E$ in $B$ is contained in $H$. Clearly, to establish the Claim, it is enough to show that all but finitely many elements of $\xi$ are contained in $\bar{E}$. Assume the contrary. Then there exists an infinite subset $C$ of $A$ such that the set $M_{\alpha}=W_{\alpha} \backslash \bar{E}$ is non-empty for each $\alpha \in C$. Then the family $\left\{M_{\alpha}: \alpha \in C\right\}$ must have an accumulation point $z$ in $B$, due to compactness of $B$. On one hand, $z$ must be in $F$, but on the other hand, $z$ cannot be in $E$, and therefore it is not in $F$, a contradiction. The Claim is established.

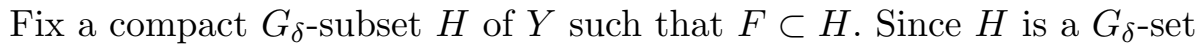
in $Y$, it follows that $H$ contains all but countably many elements of $\eta$. However, $H$ is nowhere dense in $B$, since $H \subset Y, H$ is compact, and $Y$ is nowhere locally compact. Hence, $H$ is nowhere dense in $Y$, and $H$ contains no element of $\eta$. Therefore, $\eta$ is countable, that is, the set $A$ is countable. 
TheOrem 2.4. A non-locally compact topological group $G$ is Lindelöf if and only if $G$ has a compactification $b G$ such that every compact subset $F$ of the remainder $Y=b G \backslash G$ is contained in a compact $G_{\delta}$-subset of $Y$.

Proof. The necessity follows from Henriksen-Isbell Theorem 1.1, since $G$ is the remainder of $Y$ in $b G$. It remains to prove the sufficiency.

By Theorem 1.6, it is enough to consider the following two cases.

CASE 1: $Y$ is pseudocompact. Then every compact $G_{\delta}$-subset $F$ of $Y$ has a countable base of open neighbourhoods in $Y$. Indeed, since $Y$ is Tikhonov and $F$ is compact, for every open neighbourhood $U$ of $F$ in $Y$ there is an open neighbourhood of $F$ whose closure is contained in $U$. It follows that there exists a sequence $\kappa=\left\{V_{n}: n \in \omega\right\}$ of open neighbourhoods of $F$ in $Y$ such that $\bar{V}_{n+1} \subset V_{n}$ for each $n \in \omega$, and $\bigcap \kappa=F$. Now pseudocompactness of $Y$ implies, by a standard argument, that $\kappa$ is a base of open neighbourhoods of $F$ in $Y$. Therefore, $Y$ is a space of countable type, and $G$ is Lindelöf, by the Henriksen-Isbell Theorem.

Case 2: $Y$ is Lindelöf. Then, by Theorem 1.7, $G$ is a paracompact $p$ space. By Lemma 2.3, every locally finite family of open subsets of $G$ is countable. It follows that $G$ is Lindelöf. The sufficiency is proved.

Let us say that a space $X$ is of subcountable type if every compact subset of $X$ is contained in a compact $G_{\delta}$-subset of $X$. If every compact subset of a space $X$ is a $G_{\delta}$-set in $X$, then, clearly, $X$ is of subcountable type. Now Theorem 2.4 can be reformulated as follows: a non-locally compact topological group $G$ is Lindelöf if and only if some remainder of $G$ is of subcountable type. On the other hand, it follows immediately from the Henriksen-Isbell Theorem that a non-locally compact topological group is Lindelöf if and only if every remainder of it is of countable type. Theorem 2.4 and the last statement imply the following result:

COROLlary 2.5. If $Y$ is a remainder of a topological group $G$, then the following conditions are equivalent:

(1) $Y$ is of subcountable type.

(2) $Y$ is of countable type.

Of course, in general conditions (1) and (2) are not equivalent. For example, every space with a countable network is of subcountable type, but it need not be of countable type (take a countable dense subspace of $D^{\omega_{1}}$ ).

A space $X$ is $k$-perfect if every compact subset of $X$ is a $G_{\delta}$-set. Clearly, every $k$-perfect space is of subcountable type. The next statement seems to be slightly more general.

Proposition 2.6. If a space $X$ can be mapped by a one-to-one continuous mapping onto a k-perfect space $Y$, then $X$ is of subcountable type. 
Proof. Indeed, the space $X$ in this case is itself $k$-perfect.

Corollary 2.7. If a space $X$ admits a one-to-one continuous mapping onto a perfect space, then $X$ is of subcountable type.

COROLlary 2.8. If a space $X$ admits a one-to-one continuous mapping onto a space $Y$ with a point-countable base, then $X$ is of subcountable type.

Proof. An obvious standard argument, involving A. S. Mishchenko's Theorem that every point-countable base of a compact space is countable [12], shows that every space with a point-countable base is $k$-perfect. It remains to apply Proposition 2.6.

TheOrem 2.9. A non-locally compact topological group $G$ is a Lindelöf p-space if and only if some remainder of $G$ in a compactification $b G$ is a Lindelöf space of subcountable type.

Proof. The necessity is clear, since every remainder of a Lindelöf $p$-space is a Lindelöf $p$-space (see [6]). Let us prove the sufficiency.

Put $Y=b G \backslash G$. Since $Y$ is Lindelöf and $G$ is a topological group, we conclude that $G$ is a paracompact $p$-space. Since $Y$ is of subcountable type and $G$ is not locally compact, it follows from Theorem 2.4 that $G$ is Lindelöf. Thus, $G$ is a Lindelöf $p$-space.

COROllary 2.10. If $Y$ is a remainder of a topological group $G$, then the following restrictions on $Y$ are equivalent:

(1) $Y$ is a Lindelöf space of subcountable type.

(2) $Y$ is a Lindelöf p-space.

Proof. If $G$ is locally compact, then (1) and (2) are both satisfied. If $G$ is not locally compact, then each of the conditions (1) and (2) is equivalent to the condition that $G$ is a Lindelöf $p$-space, by Theorems 2.9 and 1.2.

Now we are going to discuss certain restrictions on remainders of topological groups that guarantee that these groups are separable and metrizable. Some such conditions are already known. Here we will try to find the weakest conditions of this kind.

TheOREM 2.11. Suppose that $G$ is a topological group with a remainder $Y$ in a compactification $b G$ of $G$ such that:

(a) $Y$ is not countably compact.

(b) $Y$ is of subcountable type.

(c) Every point in $Y$ is a $G_{\delta}$-point in $Y$.

Then $G$ is separable and metrizable.

Proof. Observe that $G$ is not locally compact, since condition (a) holds. It follows from (b) and Corollary 2.5 that $Y$ is of countable type. This fact and condition (c) imply that $Y$ is first countable. Since $Y$ is not countably 
compact, we can fix a countable infinite subset $A$ of $Y$ such that $A$ is closed and discrete in $Y$. Then some $z \in G$ is an accumulation point for $A$ in $b G$, since $b G$ is compact. It follows from (a) that $G$ is nowhere locally compact. Hence, $Y$ is dense in $b G$. Therefore, $b G$ is first countable at every point of $Y$. Obviously, we can now define a countable $\pi$-base $\eta$ of $b G$ at $z$. Since $G$ is dense in $b G$, the traces of elements of $\eta$ on $G$ constitute a $\pi$-base of $G$ at $z$. Since $G$ is a topological group. it follows from Proposition 1.3 that $G$ is metrizable. By (b) and Theorem 2.4, $G$ is Lindelöf. Hence, $G$ is separable and metrizable.

COROLlary 2.12. Suppose that $G$ is a non-locally compact topological group with a Lindelof $k$-perfect remainder $Y$ in a compactification $b G$. Then $G$ is separable metrizable, and $Y$ is separable and first countable.

Proof. Clearly, $Y$ is not countably compact, since $Y$ is Lindelöf and noncompact. Thus, Theorem 2.11 is applicable, and hence $G$ is separable and metrizable. It follows that $Y$ is a Lindelöf $p$-space, hence of countable type. Also, $Y$ is of countable pseudocharacter, hence first countable. Notice that $b G$ is first countable at every point of $G$. Fix a countable dense subset $A$ of $G$. Observe that $Y$ is dense in $b G$, since $G$ is nowhere locally compact. Therefore, for each $a \in A$, we can fix a countable subset $M_{a}$ of $Y$ such that $a \in \bar{M}_{a}$. Put $M=\bigcup\left\{M_{a}: a \in A\right\}$. Clearly, $M$ is a countable dense subset of $Y$. Thus, $Y$ is separable.

COROLlarY 2.13. If $Y$ is a hereditarily Lindelöf remainder of a nonlocally compact topological group $G$, then $G$ is separable and metrizable, and $Y$ is separable and first countable.

Corollary 2.14. No L-space can be a remainder of a non-locally compact topological group.

The assumption that the group is not locally compact cannot be dropped in the above two statements. Indeed, every infinite compact space can be represented as the remainder of a discrete group by using, for example, the Aleksandrov double construction. However, consistently a hereditarily Lindelöf compactum need not be separable.

COROLlARY 2.15. If $Y$ is a perfect remainder of a topological group $G$, then every compact subspace of $Y$ has a countable base of open neighbourhoods in $Y$. In particular, $Y$ is first countable.

Proof. The remainder $Y$ is either pseudocompact or Lindelöf, since $G$ is a topological group. If $Y$ is pseudocompact, then the conclusion is clear, since $Y$ is perfect.

Let us now assume that $Y$ is Lindelöf. Then, by Corollary 2.12, $G$ is separable and metrizable. Hence, $Y$ is a space of countable type. Since $Y$ is also perfect, the required conclusion follows. 
COROLlary 2.16. Suppose that $G$ is a non-locally compact topological group with a remainder $Y$ in some compactification $b G$ of $G$ satisfying the following conditions:

$\left(\mathrm{a}_{1}\right)$ Every closed countably compact subspace of $Y$ is locally metrizable.

(b) $Y$ is of subcountable type.

Then $G$ is separable and metrizable.

Proof. First, we claim that $Y$ is not countably compact.

Assume the contrary. Then $Y$ is locally metrizable, by condition $\left(\mathrm{a}_{1}\right)$, and hence $Y$ is locally compact. However, $Y$ is dense in $b G$, since the group $G$ is not locally compact. It follows that $Y$ is open in $b G$, which implies that $G$ is not dense in $b G$, a contradiction.

Next, it follows from $\left(a_{1}\right)$ that every compact subspace of $Y$ is first countable. This fact and condition (b) imply that every point in $Y$ is a $G_{\delta}$-point. Thus, $Y$ satisfies all three conditions (a)-(c) in Theorem 2.11. Hence, $G$ is separable and metrizable.

TheOREM 2.17. Suppose that $G$ is a non-locally compact topological group with a remainder $Y$ in some compactification $b G$ of $G$ such that $Y$ has locally a $G_{\delta}$-diagonal. Then $G$ is separable and metrizable.

Proof. If $Y$ is countably compact, then $Y$ is locally metrizable, and hence locally compact, by Chaber's Theorem [12]. Then $Y$ is of subcountable type, and Corollary 2.16 is applicable. So we can assume that $Y$ is not countably compact. It is clear that every point of $Y$ is a $G_{\delta}$-point in $Y$, since $Y$ has locally a $G_{\delta}$-diagonal. Thus, $Y$ satisfies conditions (a) and (c) in Theorem 2.11.

\section{Claim. $Y$ is Lindelöf.}

By Theorem 1.6, $Y$ is either Lindelöf or pseudocompact. So we may assume that $Y$ is pseudocompact. Then $Y$ is first countable, since each point in $Y$ is a $G_{\delta}$-point. Since $Y$ is not countably compact, it follows, by a standard argument, that $G$ has a countable $\pi$-base at some point which is an accumulation point of some countable subset of $Y$. Hence, $G$ is metrizable, since $G$ is a topological group (see Proposition 1.3). Therefore, the remainder $Y$ is Lindelöf, by Theorem 1.7, since every metrizable space is a paracompact $p$-space. This proves the Claim.

To complete the proof of Theorem 2.17, it remains to establish Lemma 2.18 below.

LEMma 2.18. If $Y$ is a Lindelöf space which has locally a $G_{\delta}$-diagonal, then every compact subset $F$ of $Y$ is a $G_{\delta}$-subset of $Y$, and hence $Y$ is $k$-perfect and of subcountable type. 
Proof. By compactness of $F$ and the restriction on $Y$, there is a finite collection $\lambda=\left\{W_{1}, \ldots, W_{k}\right\}$ of open subsets of $Y$ such that each $W_{i}$ has a $G_{\delta}$-diagonal, and $F \subset \bigcup \lambda$. It is easily verified by the argument in the proof of Corollary 2.12 that the open subspace $U=\bigcup \lambda$ of $Y$ also has a $G_{\delta^{-}}$ diagonal. Since $F$ is compact and $F \subset U$, we can find an open neighbourhood $V$ of $F$ in $Y$ such that $\bar{V} \subset U$ (where the closure is taken in $Y$ ). Put $Z=\bar{V}$. Then $Z$ is a subspace of $U$, and therefore $Z$ has a $G_{\delta^{-}}$-diagonal. The subspace $Z$ is also Lindelöf, since $Z$ is closed in $Y$. Hence $Z$ admits a one-to-one continuous mapping onto a separable metrizable space $M$ [12]. Since the space $M$ is perfect, it follows that every compact subset of $Z$ is a $G_{\delta^{-}}$set in $Z$. Since $F \subset Z$, we conclude that $F$ is a $G_{\delta^{-}}$set in $Z$. But $F \subset V \subset Z$, where $V$ is open in $Y$. It follows that $F$ is a $G_{\delta}$-subset of $Y$.

Theorem 2.17 improves a result of [6]: a non-locally compact topological group is separable and metrizable if and only if it has a remainder with a $G_{\delta}$-diagonal. Local versions of some other results of similar kind can also be established with the help of Theorem 2.4. For example, it was proved in [7] that a non-locally compact topological group $G$ is separable and metrizable if and only if it has a remainder with a point-countable base. Chuan Liu observed that a local version of this theorem is true as well. Now we can easily derive this result of Chuan Liu from Theorem 2.11 and the well known Mishchenko Theorem [12] on compact spaces with a point-countable base.

After the above results had been obtained, I learned that Chuan Liu also independently proved Theorem 2.17 .

\section{On normal remainders of topological groups}

TheOREM 3.1. No Dowker space can be a remainder of a topological group.

Proof. Suppose that $Y$ is a remainder of a topological group $G$, and that $Y$ is normal. According to the Dichotomy Theorem, $Y$ is Lindelöf or pseudocompact. In the first case, $Y$ is paracompact and therefore countably paracompact, so that $Y$ is not a Dowker space. In the second case, $Y$ is pseudocompact and normal, and therefore countably compact. Hence, in the second case $Y$ is countably paracompact as well. Thus, $Y$ is not a Dowker space.

Since every pseudocompact normal space is countably compact, it is natural to ask when a topological group $G$ has a normal remainder. In this direction, we have the following observations. The next result follows immediately from Theorem 1.6 and the above remark.

COROLlaRY 3.2. If some remainder of a topological group $G$ is normal, then either every remainder of $G$ is countably compact, or every remainder of $G$ is Lindelöf. 
Proof. Suppose that some remainder of $G$ is not Lindelöf. Then it follows from Theorem 1.6 that all remainders of $G$ are pseudocompact. Fix a normal remainder $Y$ of $G$. Then $Y$ is countably compact, since every pseudocompact normal space is countably compact [12]. However, countable compactness is preserved by perfect mappings in both directions. Every remainder of a Tikhonov space $X$ is an image under a perfect mapping of the StoneCech remainder of $X$ [12]. Therefore, every remainder of $G$ is countably compact.

COROLlARY 3.3. If some remainder of a topological group $G$ is normal, then the extent of every remainder $Y$ of $G$ is countable, that is, every closed discrete subset of $Y$ is countable.

Proof. By Corollary 3.2, the space $Y$ is countably compact or Lindelöf. Clearly, in either case the extent of $Y$ is countable.

COROLlary 3.4. If some remainder $Y$ of a topological group $G$ is normal, then $Y$ is collectionwise normal.

Let us now consider when a renainder of a topological group is perfectly normal. A partial answer is given by the following somewhat unexpected theorem. Recall that $M A+\neg C H$ stands for Martin's Axiom combined with the negation of the Continuum Hypothesis.

Theorem 3.5. Assume $M A+\neg C H$. Then a remainder $Y$ of a topological group $G$ is perfectly normal if and only if $Y$ is hereditarily Lindelöf.

Proof. If $Y$ is hereditarily Lindelöf, then $Y$ is perfectly normal.

Let us now assume that $Y$ is perfectly normal. By the Dichotomy Theorem, it suffices to consider the following two cases.

Case 1: $Y$ is Lindelöf. Then $Y$ is hereditarily Lindelöf, since $Y$ is perfect.

CASE 2: $Y$ is pseudocompact. Then $Y$ is countably compact, since $Y$ is normal. However, under $M A+\neg C H$ every countably compact perfectly normal space is compact [14]. It follows that $Y$ is hereditarily Lindelöf in this case as well.

We can strengthen the necessity in Theorem 3.5 as follows:

THEOREM 3.6. Let $G$ be a topological group with a $k$-perfect remain$\operatorname{der} Y$. Then $Y$ is first countable.

Proof. It follows from Corollary 2.5 that $Y$ is of countable type. Therefore, $Y$ is first countable, since every point in $Y$ is a $G_{\delta}$-set.

Problem 3.7. Find restrictions on a topological group $G$, other than that $G$ is a paracompact p-space, which guarantee that $G$ has a normal remainder. 
Problem 3.8. Is a normal remainder of a topological group always Lindelöf?

PROBLEM 3.9. Is perfect normality of a remainder of a topological group equivalent to hereditary Lindelöfness of this remainder (in ZFC)?

Here is a curious application of Corollary 3.2 to embeddings:

TheOREM 3.10. Suppose that $G$ is a dense subgroup of a sequential topological group $H$. Suppose also that some remainder of $G$ is normal. Then either $H$ is metrizable or $G=H$.

Proof. Take a compactification $B$ of $H$. Then $B=b G$ is also a compactification of $G$, and $G \subset H \subset b G$. Put $Y=b G \backslash G$ and $A=H \backslash G$. Assume that $G \neq H$. Then $A \neq \emptyset$, and $G$ is not open in $H$, since every open dense subgroup of a topological group coincides with the group [15], [11]. Hence, $A$ is not closed in $H$, and some sequence in $A$ converges to a point in $G=H \backslash A$. Since $A \subset Y$, it follows that $Y$ is not countably compact. Now Corollary 3.2 implies that every remainder of $G$ is Lindelöf. It follows that $G$ is a paracompact $p$-space. The tightness of $G$ is countable, since the space $G$ is sequential. However, if a topological group of countable tightness is a paracompact $p$-space, then it is metrizable [3], [11]. Therefore, $G$ is metrizable. Since $G$ is dense in $H$ and $H$ is a topological group, it follows that $H$ is metrizable as well.

ExAmple 3.11. Let $D$ be the discrete topological group $\{0,1\}$. Put $B=$ $D^{\tau}$, where $\tau$ is an uncountable cardinal number. Let $G$ be the $\sigma$-product and $H$ be the $\Sigma$-product of $\tau$ copies of the group $D$ (over zero-point). Then $G$ and $H$ are dense subgroups of the topological group $B$, and $G \subset H$. Moreover, the space $H$ is Frechét-Urysohn (see [12]).

Since $G \neq H$ and $H$ is not metrizable, it follows from Theorem 3.10 that the remainder $Y=B \backslash G$ is not normal. Clearly, $Y$ is not countably compact, though $Y$ is pseudocompact, by Theorems 1.6 and 1.7.

4. Some observations on topological groups with homeomorphic remainders. In this section we will discuss another general situation. One might consider the following general question: how close must be the properties of topological groups $G$ and $H$ if they have homeomorphic compactifications? This is, probably, not a very good question, since such groups $G$ and $H$ may differ drastically in their topological properties, as the next example shows.

EXAMPLE 4.1. Let $B$ be an infinite compact metrizable topological group, $M$ be a countable dense subgroup of $B$, and $H=M^{\tau}$, where $\tau$ is some uncountable cardinal number. Furthermore, let $K$ be the $\Sigma$-product of $\tau$ copies of $B$, and $L=B^{\tau} \times M^{\omega}$. Finally, put $G=B^{\tau}$. Then $H, K, L, G$ 
are topological groups, and $G=B^{\tau}$ is a compactification of each of them. However, any two of these groups differ much in their properties, and the same is true for their remainders in $G$.

Motivated by this example, we take another approach and formulate a different question:

Problem 4.2. Suppose that some remainders of topological groups $G$ and $H$ are homeomorphic. Find topological properties which are shared by $G$ and $H$.

Let us call topological groups $G$ and $H$ r-equivalent if some remainders of $G$ and $H$ are homeomorphic.

Obviously, the relation of $r$-equivalence is symmetric and reflexive.

COROLlary 4.3. Suppose that topological groups $G$ and $H$ are $r$-equivalent. Then:

(1) If $G$ is a paracompact p-space, then $H$ is a paracompact p-space.

(2) If $G$ is a Lindelöf p-space, then $H$ is a Lindelöf p-space.

(3) If $G$ is Lindelöf, then $H$ is Lindelöf as well.

Proof. If $G$ is locally compact, then all three statements are trivially true. So we may assume that neither $G$ nor $H$ is locally compact. Statement (1) follows from Theorem 1.7. Statement (2) follows from the characterization of Lindelöf $p$-spaces in terms of remainders (Theorem 1.2). Statement (3) follows from Theorem 1.1.

Corollary 4.3 is complemented by the following two statements the proofs of which are trivial.

Proposition 4.4. Suppose that topological groups $G$ and $H$ are $r$-equivalent. Then:

(4) If $G$ is locally compact, then $H$ is locally compact.

(5) If $G$ is Čech-complete, then $H$ is Čech-complete.

(6) If $G$ is compact, then $H$ is also compact.

Proposition 4.5. Any two compact topological groups are $r$-equivalent, and any two locally compact non-compact topological groups are $r$-equivalent.

However, one may have noticed that neither the class of separable metrizable groups, nor the class of metrizable groups is included in the above lists. To prove that the class of separable metrizable groups is $r$-invariant, we need a characterization of separable metrizable groups by a property of an arbitrary remainder of such a group. Here it is, an almost obvious one: 
Proposition 4.6.

(a) If a non-locally compact topological group $G$ is separable and metrizable, then every remainder of $G$ has a countable $\pi$-base.

(b) If some remainder of a non-locally compact topological group $G$ has a countable $\pi$-base, then $G$ is separable and metrizable.

Proof. (a) Suppose that $\eta$ is a countable base of $G$, and that $b G$ is an arbitrary compactification of $G$. Put $Y=b G \backslash G$, and, for each $V \in \eta$, fix an open subset $U_{V}$ of $b G$ such that $V=U_{V} \cap G$. Then the family $\xi=\left\{U_{V} \cap Y: V \in \eta\right\}$ is, clearly, a countable $\pi$-base of $Y$.

(b) Fix a compactification $b G$ of $G$ such that the remainder $Y=b G \backslash G$ has a countable $\pi$-base $\gamma$. For each $V \in \gamma$ fix an open subset $U_{V}$ in $b G$ such that $U_{V} \cap Y=V$. Then the family $\xi=\left\{U_{V} \cap G: V \in \gamma\right\}$ is a countable $\pi$-base of the space $G$. Since $G$ is a topological group, it follows that $G$ is separable and metrizable (see Proposition 1.3 or [2]).

THEOREM 4.7. If a non-locally compact topological group $G$ is $r$-equivalent to a topological group $H$, and $G$ is separable and metrizable, then $H$ is also separable and metrizable.

Proof. By Proposition 4.4, $H$ is also non-locally compact. Now it follows from Proposition 4.6 that $H$ is separable and metrizable.

Proposition 4.5 shows that the assumption that the groups under consideration in Proposition 4.6 and Theorem 4.7 are non-locally compact is essential.

The case of topological groups $r$-equivalent to metrizable groups is more special, since in this case we do not have a result similar to Proposition 4.6. Instead, we have the following statement:

Theorem 4.8. A non-locally compact topological group $G$ is metrizable if and only if some remainder $Y$ of $G$ is a non-countably compact space of countable $\pi$-character.

Proof. Suppose that $G$ is metrizable. Then there exists a compactification $b G$ of $G$ such that $b G$ is an Eberlein compactum [4]. However, every Eberlein compactum is a space of countable tightness and has a countable $\pi$-base at each point (see [4]). Since $G$ is nowhere locally compact, the remainder $Y=b G \backslash G$ is dense in $b G$. Therefore, $Y$ also has a countable $\pi$-base at each point. Observe that the space $b G$ is first countable at each point of $G$, since $G$ is metrizable and dense in $b G$. It follows that some sequence in $Y$ converges to an element of $G$. Hence, $Y$ is not countably compact. The necessity is proved.

The proof of the sufficiency should be clear by now: we can easily construct a countable $\pi$-base at some point of $G$ belonging to the closure of a countable subset of the remainder. 
Problem 4.9. Is metrizability preserved by r-equivalence in the class of non-locally compact topological groups?

Acknowledgements. The author is grateful to the referee for careful refereeing of this paper and many helpful comments.

\section{References}

[1] A. V. Arhangel'skii, On a class of spaces containing all metric and all locally compact spaces, Mat. Sb. 67 (109) (1965), 55-88 (in Russian); English transl.: Amer. Math. Soc. Transl. 92 (1970), 1-39.

[2] - Relations between invariants of topological groups and their subspaces, Russian Math. Surveys 35 (1980), no. 3, 1-23.

[3] -, Classes of topological groups, ibid. 36 (1981), 151-174.

[4] -, Topological Function Spaces, Kluwer, 1992.

[5] - Some connections between properties of topological groups and of their remainders, Moscow Univ. Math. Bull. 54 (1999), no. 3, 1-6.

[6] - Remainders in compactifications and generalized metrizability properties, Topology Appl. 150 (2005), 79-90.

[7] —, More on remainders close to metrizable spaces, ibid. 154 (2007), 1084-1088.

[8] -, Two types of remainders of topological groups, Comment. Math. Univ. Carolin. (2008).

[9] —, Remainders of topological groups and of their subspaces, C. R. Acad. Sci. Bulg. 61 (2008), 5-7.

[10] A. V. Arhangel'skii and V. I. Ponomarev, Fundamentals of General Topology in Problems and Exercises, Reidel, 1984 (translated from Russian).

[11] A. V. Arhangel'skii and M. G. Tkachenko, Topological Groups and Related Structures, Atlantis Press and World Sci., 2008.

[12] R. Engelking, General Topology, PWN, Warszawa, 1977.

[13] M. Henriksen and J. R. Isbell, Some properties of compactifications, Duke Math. J. 25 (1958), 83-106.

[14] I. Juhász, Consistency results in topology, in: Handbook of Mathematical Logic, Part B: Set Theory, J. Barwise (ed.), Stud. Logic Found. Math. 90, North-Holland, Amsterdam, 1977, 503-522.

[15] W. Roelke and S. Dierolf, Uniform Structures on Topological Groups and their Quotients, McGraw-Hill, New York, 1981.

Department of Mathematics

Ohio University

Athens, OH 45701, U.S.A.

E-mail: arhangel@math.ohiou.edu

Received 24 December 2007;

in revised form 9 January 2009 